\title{
Vertragsfreiheit und Inhaltskontrolle von Eheverträgen
}

Dass sich in der Grundsatzentscheidung des BGH zur Inhaltskontrolle von Eheverträgen keine Spur des »dramatischen und engagierten Ringens um Reichweite und Grenzen der Ehevertragsfreiheit « findet, ${ }^{1}$ fördert zwar die praktische Umsetzbarkeit dieser Entscheidung, die weit über den zu beurteilenden Fall hinaus allgemeine Grundsätze für die künftige Kontrolle von Eheverträgen erarbeitet. Gleichwohl tut eine Standortbestimmung Not, um aus dem grundrechtlichen Schutzauftrag und dem Blick auf die bisherige Entwicklung des Instruments der Inhaltskontrolle Antworten auf die vielen noch offenen Fragen ${ }^{2}$ der künftigen Inhaltskontrolle von Eheverträgen zu finden. Hierbei muss die Diskussion auch wieder die Fragen der Parität der Vertragsschließenden einbeziehen. ${ }^{3}$

\section{Vertragsfreiheit und Inhaltskontrolle}

\section{Vertragsfreiheit und Vertragsgerechtigkeit}

Vertragsfreiheit ist aus der Sicht der Zivilrechtslehre ein wichtiges Strukturelement ${ }^{4}$ der freiheitlichen Wirtschafts- und Rechtsordnung. Sie basiert auf der liberalen Vertragstheorie, welche sich das BGB noch ${ }^{5}$ zu eigen gemacht hat ${ }^{6}$ (»from status to contract $\aleph^{7}$ ). Bald setzte jedoch eine Gegenbewegung ein, welche die Vertragsfreiheit unter das Gebot der Vertragsgerechtigkeit stellen wollte. ${ }^{8}$ Man sah das »ewige Dilemma

1 So die Kritik von Dauner-Lieb, FF 2004, 65 am Grundsatzurteil BGH, NJW 2004, 930 f. = FamRZ 2004, 601 ff. = DNotZ 2004, 550 ff. = ZNotP 2004, 157 ff. mit Stellungnahme $C$. Münch, ZNotP 2004, 122 ff. Eine weitere Grundsatzentscheidung des BGH ist inzwischen zur Ausübungskontrolle ergangen. Der BGH will eine Vertragsänderung nur bis zum Ausgleich der ehebedingten Nachteile, BGH, NJW 2005, 139 f. hierzu C. Münch, FamRZ 2005, 570 ff. Vgl. inzwischen auch BGH v. 25.5.05 (XII ZR 296/01).

2 Vgl. die Überschrift von Rakete-Dombek, NJW 2004, 1273 ff: »Nach dem Urteil ist vor dem Urteil «.

3 So die ausdrückliche Forderung von Hohmann-Dennhardt, FF 2004, 233, 235; vgl. zur Parität bei beurkundeten Verträgen C. Münch, DNotZ 2004, $901 \mathrm{ff}$.

4 Fastrich, Richterliche Inhaltskontrolle im Privatrecht, 3; Flume, Festschrift Hundert Jahre Deutsches Rechtsleben, Band I, 1960, 135, 141; Hönn, Kompensation gestörter Vertragsparität, 1982, 5; Raiser, JZ 1958, 1 ff.; Singer, Selbstbestimmung und Verkehrsschutz im Recht der Willenserklärungen, 1995, 1.

5 Hönn (FN 4), 5 ff.; M. Wolf, Rechtsgeschäftliche Entscheidungsfreiheit, 1970, 8 ff.; Radbruch, Einführung in die Rechtswissenschaft, 13. Aufl., 1980, 103; zur Historisierung der Vertragsfreiheit Abegg, KritV 2004, 197 ff.

6 Raiser, JZ 1958, 1, 2; zur europäischen Dimension der Vertragsfreiheit: Kissel in Festschrift für Alfred Söllner, 2000, $491 \mathrm{ff}$.

7 So die berühmt gewordene Formel von Maine, Ancient Law, 1861, 141; vgl. hierzu Schwab, From Status to Contract ?, DNotZ-Sonderheft 2001, 9 ff.

8 Raiser, JZ 1958, 1, 3. 
der Privatautonomie, dass sie immer wieder durch ungleiche Machtverteilung in Frage gestellt ist «. ${ }^{9}$ Der Schutz des Schwächeren wurde zum eigenen zivilrechtlichen Thema. ${ }^{10} \mathrm{Ob}$ der Primat nun bei der Vertragsgerechtigkeit ${ }^{11}$ im Einzelfall oder aber bei der Vertragsfreiheit ${ }^{12}$ und der Rechtssicherheit als Bestandteil des rechtsstaatlichen Prinzips nach Art. 20 Abs. 3 GG $^{13}$ liegt, das blieb bis heute - auch rechtspolitisch - umstritten.

\section{Zivilrechtliche Vertragstheorien}

Eine Reihe von Vertragstheorien versuchte in der Folge, die Vertragsfreiheit näher zu begründen. Die prägendste stammt von Schmidt-Rimpler. ${ }^{14}$ Seine Theorie der Richtigkeitsgewähr besagt, dass beim Vertrag jeder Partner die Rechtsfolgen aus seiner Sicht prüft und daher der von beiden mit freiem Willen abgeschlossene Vertrag durch diesen Vertragsmechanismus zur Richtigkeit führt. Nach Schmidt-Rimpler leistet der Vertrag dies nicht mehr, »wenn die Freiheit der Entscheidung typisch, insbesondere wegen Abhängigkeit einer Partei von der anderen oder Unterlegenheit in der Wertungsfähigkeit, fehlt «. ${ }^{15}$ Weitere wichtige Vertragstheorien stammen von Flume, ${ }^{16}$ Manfred Wolf, ${ }^{17}$ Hönn ${ }^{18}$ und Raiser. ${ }^{19}$

9 Flume (FN 4), 135, 143.

10 Kramer, Die Krise des liberalen Vertragsdenkens, 1974; Lieb, DNotZ 1989, 274, 276; Weitnauer, Der Schutz des Schwächeren im Zivilrecht, 1975; von Hippel Eike, Der Schutz des Schwächeren, 1982. Schon früh bezeichnet Otto von Gierke, Die sociale Aufgabe des Privatrechts, 1889, 28 f. die Vertragsfreiheit als »eine furchtbare Waffe in der Hand des Starken, ein stumpfes Werkzeug in der Hand des Schwachen«, so dass sie zum »Mittel der Unterdrückung des einen durch den anderen, der schonungslosen Ausbeutung geistiger und wirtschaftlicher Übermacht« werde. Einen kurzen Abriss historischer Stellungnahmen gibt Zöllner, AcP 196 (1996), 1, 16 ff.

11 So etwa Ihering, Recht und Sitte, 1924, 131: „Es gibt in meinen Augen keinen verhängnisvolleren Irrtum, als dass der Vertrag als solcher, sofern sein Inhalt nur nicht gesetzwidrig oder unmoralisch sei, einen gerechtfertigten Anspruch auf Schutz des Gesetzes habe. ... Das Interesse der Gesellschaft aber geht auf das, was nicht bloß dem einzelnen, sondern was allen passt, bei dem alle bestehen können, und das ist ... nichts anderes als die Gerechtigkeit. Sie steht über der Freiheit.«

12 So etwa Langenfeld, Vertragsgestaltung, 3. Aufl., 2004, Rdz. 372.

13 Hierzu C. Münch, NJW 1996, 3320 f. und C. Münch, Festschrift für Hugo J. Hahn, 1997, 673 ff.

14 Schmidt-Rimpler, AcP 147 (1941), $130 \mathrm{ff}$.

15 Schmidt-Rimpler, AcP 147 (1941), 130, 158, FN 34.

16 »Selbstbestimmungstheorie «: Selbstbestimmung und ihre Anerkennung durch die Rechtsordnung bilden zusammen die Voraussetzungen für die Gültigkeit eines Rechtsgeschäftes; die Vertragsfreiheit erfordert danach begrifflich die Rechtsordnung als Korrelat. Ferner muss tatsächlich die Macht zur Selbstbestimmung bestehen (Flume FN 4, 135, 143).

17 Danach $(M$. Wolf, FN 5, 27) ist die rechtsgeschäftliche Entscheidungsfreiheit Voraussetzung der Gültigkeit von Willenserklärungen, nicht erst ihr Fehlen Schranke der Rechtsausübung (Postulat einer Art wirtschaftlicher Geschäftsfähigkeit).

18 Parität wird rein formal definiert als die vom positiven Recht den Vertragsparteien eingeräumte Rechtsstellung, welche ihnen einen Interessenausgleich in Selbstbestimmung mit der Chance der Äquivalenz eröffnet (Hönn, FN 4).

19 Dem Vertrag wird wegen seiner Bedeutung für die Gesamtwirtschaft und die Gesellschaft Anerkennung und Rechtsschutz gewährt. Er basiert auf der Selbstbestimmung der Beteilig- 


\section{Inhaltskontrolle als Schutzmechanismus}

Auf dieser Grundlage entwickelten Gesetzgebung und Rechtsprechung verschiedene Schutzmechanismen, die in den jeweiligen Rechtsbereichen mit eigenen Mitteln punktuell der Vertragsgerechtigkeit zum Durchbruch verhelfen sollen: ${ }^{20}$

- Leitbildabweichungskontrolle bei AGBs zunächst durch richterliche Inhaltskontrolle, ${ }^{21}$ seit 1976 durch das AGBG und heute durch $\S \S 305$ ff. BGB;

- Schriftformerfordernis, zwingende Vertragsinhalte und Widerrufsrechte im Abzahlungsgesetz (1894 !), später Verbraucherkreditgesetz und jetzt Verbraucherdarlehen nach $\S \S 491 \mathrm{ff}$. BGB ${ }^{22}$

- Unterrichtungspflichten und Widerrufsrechte im Fernabsatzgesetz - nun $\S \S 312 \mathrm{~b}$ ff. BGB - oder Haustürwiderrufsgesetz - nun $\S \S 312$ ff. BGB - sowie - § 312e BGB für den elektronischen Geschäftsverkehr; ${ }^{23}$

- Mieterschutzvorschriften durch zwingenden Preis- und Bestandsschutz im Wohnungsmietrecht, ${ }^{24}$ ergänzend zusätzlich richterliche Inhaltskontrolle $;^{25}$

ten im herrschaftsfreien Raum. Dabei will Raiser den Vertrag nicht erst bei Missbrauch an der Sittenwidrigkeit scheitern lassen, sondern plädiert für eine schon vorher erfolgende richterliche Kontrolle der ordnungsgemäßen Verwendung des Rechtsinstituts Vertrag bei intellektueller Unterlegenheit (Raiser, Festschrift Hundert Jahre Deutsches Rechtsleben, Band I, 1960, 101 ff., 104, 119, 131; Raiser, JZ 1958, 1, 7).

20 Kritisch zu diesem punktuellen Lösungsansatz und zur kaum verbesserten Erkenntnissituation seit Erlass des BGB: Limbach, KritV 1986, 165 und 178.

21 »Da Allgemeine Geschäftsbedingungen ihre Rechtswirksamkeit nicht von einer (nicht bestehenden) Privatautonomie, sondern nur von der Unterwerfung des anderen Vertragsteiles ableiten können, muss ihnen die Anerkennung versagt werden, soweit die von ihnen für eine unbestimmte Anzahl von Einzelfällen aufgestellte Regel mit den Grundsätzen von Treu und Glauben nicht zu vereinbaren sind. Soweit Vorschriften des dispositiven Rechtes ihre Entstehung nicht nur Zweckmäßigkeitserwägungen, sondern einem aus der Natur der Sache sich ergebenden Gerechtigkeitsgebot verdanken, müssen bei einer abweichenden Regelung durch Allgemeine Geschäftsbedingungen Gründe vorliegen, die für die von ihnen zu regelnden Fälle das dem dispositiven Recht zugrundeliegende Gerechtigkeitsgebot in Frage stellen und eine abweichende Regelung als mit Recht und Billigkeit vereinbar erscheinen lassen. « So BGHZ 41,151, 154.

22 Ein eindrucksvoller Beleg des Schutzes des Schwächeren findet sich in diesem Bereich aktuell in dem Wandel der Rechtsprechung des BGH zur Rückabwicklung eines kreditfinanzierten Beitritts zu einem geschlossenen Immobilienfonds. Nach langer Rechtsprechungsgeschichte gelangt der BGH nun zum Ergebnis, dass bei Nichtigkeit des Darlehensvertrags wegen Verstoßes gegen das Rechtsberatungsgesetz der Anleger der Bank nicht Rückzahlung des Darlehens, sondern nur Übertragung des (wertlosen) Fonds-Anteils schulde, vgl. BGH, DStR 2004, 1345 ff.

23 Diese sind in der Verordnung über Informations- und Nachweispflichten nach bürgerlichem Recht auf der Grundlage der Art. 238 ff. EGBGB für die Bereiche Fernabsatz, Teilzeit-Wohnrechte, elektronischer Geschäftsverkehr und Reisevertrag detailliert festgelegt, BGBl. 2002 I, $3002 \mathrm{ff}$.

24 Zur Geschichte des sozialen Mietrechts: Honsell, AcP 186, 115 ff.

25 Zusammenstellung bei Fastrich (FN 4), 109 ff. 
- Richterliche Inhaltskontrolle gegenüber formelhaften Gewährleistungsausschlüssen beim Erwerb neu errichteter oder noch zu errichtender Eigentumswohnungen und Häuser in notariellen Verträgen; ${ }^{26}$

- Richterliche Inhaltskontrolle bei der Publikums $\mathrm{GmbH} \& \mathrm{Co}^{27}$ sowie allgemein bei Kündigungsklauseln, Geschäftsführungsmaßnahmen oder Beschlüssen;

- Billigkeitsprüfung auf der Grundlage des $§ 315$ BGB im Arbeitsrecht ${ }^{28}$ wegen des einseitigen Direktionsrechts des Arbeitgebers. ${ }^{29}$

Insgesamt also ein buntes Bild der Inhaltskontrolle, Ausübungs- und Billigkeitskontrolle durch Gerichte, gesetzlicher Informationsmodelle ${ }^{30}$ und gesetzlicher Angemessenheitsprüfung, welche entweder die Ausübung bestimmter Rechte untersagt oder einzelne Klauseln und Vertragsbestimmungen für unwirksam erklärt. In diesen Zusammenhang gehören auch Gesetze auf dem Gebiet des Wettbewerbs- und Kartellrechts, welche die Marktmacht eines Vertragsteils zugunsten ausgewogener Verhandlungsmacht zurückdrängen wollen. Inhaltskontrolle kann in diesem Sinne als erweiterter Begriff verstanden werden, der verschiedene Methoden zum Schutz des Schwächeren kennzeichnet.

Vor der Komplexität der Funktionszusammenhänge ${ }^{31}$ versagt bisher eine einheitliche Dogmatik der Inhaltskontrolle. Ungeklärt ist vor allem das Verhältnis zur Sittenwidrigkeitsprüfung nach $\S 138$ BGB, sind doch die meisten Stimmen der Auffassung, die Inhaltskontrolle müsse deutlich vor den Grenzen des $\S 138$ BGB ansetzen ${ }^{32}$ und dürfe andererseits keine Einzelfallprüfung sein, sondern eine Untersuchung typisierbarer Fallgestaltungen. ${ }^{33}$

\section{Entwicklung im Familienrecht}

Im Familienrecht verlief die Entwicklung gegenläufig oder zeitversetzt. Zunächst war die Ehe als vorgegebene Institution einer vertraglichen Regelung unzugänglich. ${ }^{34}$ Le-

26 BGHZ 62, 251; BGHZ 65, 359; BGHZ 74, 204 = DNotZ 1979, 741 = NJW 1979, 1406; BGH, NJW 1982, 2243 = DNotZ 1982, 626; BGH, DNotZ 1984, 760 mit Anm. Stürner; BGHZ 101, 350 f.; hierzu Ulmer, DNotZ 1982, 587 ff.

27 BGHZ 64, 238 ff.; BGH, NJW 1977, 2263.

28 Vgl. etwa BAG, DB 1986, 132; BAG, DB 1992, 1143. Zum Thema: Fastrich (FN 4), 159 ff.; Preis, Grundfragen der Vertragsgestaltung im Arbeitsrecht, 1993, 216 ff.

29 Insbesondere zu dieser arbeitsrechtlichen Thematik von Stebut, Der soziale Schutz als Regelungsproblem des Vertragsrechts, 1982.

30 So die Bezeichnung bei Dauner-Lieb, Verbraucherschutz durch Ausbildung eines Sonderprivatrechts für Verbraucher - Systemkonforme Weiterentwicklung oder Schrittmacher für Systemveränderung ?, 1983, $69 \mathrm{ff}$.

31 Fastrich (FN 4), 278.

32 Fastrich (FN 4), 17 ff.; Kramer (FN 10), 51; von Westphalen, MDR 1994, $5,8$.

33 Coester-Waltjen, AcP 190 (1990), 1, 27; Fastrich (FN 7), 224 ff.; ausführlich Lieb, AcP 178 (1978), 196, 221; Spieß, DVBl. 1994, 1222, 1228; Wellenhofer-Klein, ZIP 1997, 775, 780.

34 Schmidt-Rimpler, AcP 147 (1941), 130, 150; Schwab, DNotZ-Sonderheft 2001, 9/10; Stoll in Nipperdey, Die Grundrechte und Grundpflichten der Reichsverfassung, Band III, 1930, Art. 152, 180 (einschließlich des Ehegüterrechts). 
diglich in Bezug auf die vermögensrechtlichen Verhältnisse sollte Vertragsfreiheit zugelassen sein. Dementsprechend umfasst die Legaldefinition des BGB beim Ehevertrag nur den Vertrag über die güterrechtlichen Verhältnisse ${ }^{35}$ und bricht so mit einer langen ehevertraglichen Tradition der Festlegung personaler Elemente. ${ }^{36}$ Erst die 1. Eherechtsreform 1976 verzichtete auf ein bestimmtes Ehemodell. Die Ehe wurde »Gefäß ohne Inhalt « ${ }^{37}$ und damit vertraglicher Regelung zugänglich. ${ }^{38}$ Vertragsfreiheit gewann ab hier Bedeutung im Familienrecht.

\section{Die beiden Komponenten der Vertragsfreiheit}

Hervorgehoben sei, dass die Vertragsfreiheit aus zwei Komponenten besteht:

- Freiheit der Vertragsparteien, mit einem beliebigen Partner Verträge selbst gewählten Inhalts zu schließen.

- Einbindung in die Rechtsordnung, die Schutz gegen Vertragsverletzungen durch Rechtszwang gewährt, weil im Vertrag grundsätzlich die Richtigkeitschance ruht. Insoweit ist Vertragsfreiheit normativ konstituierte Freiheit, ${ }^{39}$ enthält der Vertrag Elemente einer objektiven Ordnung. ${ }^{40}$

Diesen Schutz stellt der Staat aber da in Frage, wo die Richtigkeitschance nicht besteht; hier setzt das soeben aufgezeigte Schutzinstrumentarium an. Auch das BVerfG äußert sich dahin, die Privatautonomie sei notwendig auf staatliche Durchsetzung angewiesen. Ihre Gewährleistung denke die justitielle Realisierung gleichsam mit. ${ }^{41}$ Diese zweite Komponente bietet sich als Nahtstelle für den Blick auf die Diskussion der Vertragsfreiheit im Verfassungsrecht an.

\section{Die Bedeutung der Grundrechte für die Vertragsfreiheit}

Die Einwirkung von Grundrechten auf das Privatrecht wird noch immer streitig diskutiert. ${ }^{42}$ Sie wirkt sich nun auch auf die Inhaltskontrolle von Verträgen aus.

\section{Von der Drittwirkung zur Schutzpflicht}

Nach der Lehre von der unmittelbaren Drittwirkung ${ }^{43}$ ist nicht nur der Staat Adressat der Grundrechte in ihrer Funktion als Abwehrrecht, sondern auch Privatrechtssubjekte

$35 \S 1432$ BGB a. F., jetzt $\S 1408$ BGB. Eingehend hierzu Grziwotz, DNotZ-Sonderheft 1998, $228,240$.

36 Hattenhauer, FamRZ 1989, 225, 229.

37 Schwab in Staatslexikon, Hrsg. Görres-Gesellschaft, 7. Aufl., 1986, Band 2, Sp. 120; Grziwotz, DNotZ-Sonderheft 1998, 228, 249 ff.

38 Schwab, DNotZ-Sonderheft 1977, 51 ff.; vgl. auch Willoweit, JuS 1977, 292, 296.

39 Höfling, Vertragsfreiheit, 1991, 22, 27.

40 Fastrich (FN 4), 53.

41 BVerfG, NJW 1994, 36, 38.

42 So wird erst jüngst die Frage erhoben: »Grundrechtstotalitarismus oder Selbstbehauptung des Zivilrechts? « Simon, AcP 2004, 264 ff.

43 Vgl. etwa Nipperdey, Grundrechte und Privatrecht, 1961, 15; Hager, JZ 1994, 373 ff. 
sind unmittelbare Adressaten der Grundrechte. Demgegenüber binden nach der Lehre von der mittelbaren Drittwirkung die Grundrechte unmittelbar nur den Staat. Sie erlangen jedoch mittelbar Auswirkung auf das Privatrecht durch das Medium der Privatrechtsnormen, insbesondere der Generalklauseln und führen zu einer grundrechtsgeleiteten Interpretation ${ }^{44}$ des Privatrechts. ${ }^{45}$ Die Grundrechte sind insoweit zugleich objektive Wertordnung und verfassungsrechtliche Grundentscheidung für alle Bereiche des Rechts. ${ }^{46}$

Mehr und mehr in den Vordergrund der Argumentation tritt aber die vom BVerfG entwickelte ${ }^{47}$ und von Canaris $^{48}$ ausführlich begründete Schutzgebotsfunktion der Grundrechte. Adressat dieser Schutzpflicht ist der Staat, d.h. in erster Linie der Gesetzgeber und dann der Richter. Das Fehlen von Schutzvorschriften kann verfassungswidrig sein, wenn damit das verfassungsrechtlich gebotene Schutzminimum unterschritten wird (Untermaßverbot) ${ }^{49}$ Insoweit liegt ein Verfassungsverstoß des Gesetzgebers vor. Der Richter hat diese Einwirkung der Grundrechte auf das Privatrecht zu prüfen, sonst stellt sein Urteil eine Grundrechtsverletzung durch die öffentliche Hand dar. Die Grundrechte richten sich also danach nicht gegen den Privaten, sondern nur gegen den Staat. ${ }^{50}$ Diese Ansicht erlaubt aber mittelbar die Inhaltskontrolle von Rechtsgeschäften zwischen Privaten am Maßstab der Grundrechte.

\section{Entscheidungen des BVerfG zur Inhaltskontrolle}

Diesen Schutzauftrag der Grundrechte hat das BVerfG gerade auch im Hinblick auf die Vertragsfreiheit angewandt und damit der Diskussion um die Inhaltskontrolle von Verträgen eine völlig neue Richtung gegeben.

Dies geschah erstmals mit der sog. Handelsvertreterentscheidung im Jahre $1990 .^{51}$ Darin erkannte das BVerfG zunächst die Privatautonomie als grundrechtlich geschützte Position an und setzt dann entlang der zivilrechtlichen Vertragstheorien die Argumentation damit fort, die Privatautonomie beruhe auf dem Prinzip der Selbstbestimmung und setze somit voraus, dass diese Selbstbestimmung tatsächlich gegeben sei. Habe eine Vertragspartei ein so starkes Verhandlungsübergewicht - gestörte Vertragsparität -, dass sie die vertraglichen Regelungen faktisch einseitig bestimmen könne, so sei Fremd-, nicht Selbstbestimmung gegeben. Wenn dabei auf der fremdbestimmten Seite über grundrechtlich verbürgte Positionen verfügt werde, dann greife der Schutzauftrag der Verfassung ein. ${ }^{52}$ Diese Linie wurde fortgesetzt mit dem Urteil

44 BVerfGE 99, 185, 196; vgl. Hermes, VVDStRL 61, 119, 121: »interpretationsleitende« Berücksichtigung der Grundrechte.

45 Grundlegend BVerfGE 7, 198 ff. (Lüth).

46 BVerfGE 7, $198 \mathrm{ff}$.

47 BVerfGE 39, 1 ff. (Reform $§ 218$ StGB).

48 Canaris, AcP 184, $201 \mathrm{ff}$.

49 Canaris, AcP 184, 201, 228; Fastrich (FN 4), 76.

50 Rüfner, Gedächtnisschrift für W. Martens, 1987, 215, 220.

51 BVerfGE 81, 242 ff. = NJW 1990, $1469 \mathrm{f}$.

52 Dieser richte sich zunächst an den Gesetzgeber, der den Grundrechtsschutz zu sichern habe. Die Anwendung und Konkretisierung der Gesetze obliegt dem Schutzauftrag des Richters. 
des BVerfG zu ruinösen Familienbürgschaften. ${ }^{53}$ Hier betont das Gericht aber zugleich, die grundgesetzlich geschützte Privatautonomie verpflichte den Gesetzgeber, rechtsgeschäftliche Gestaltungsmittel zur Verfügung zu stellen, die als rechtsverbindlich zu behandeln sind und auch im Streitfall durchsetzbare Rechtspositionen begründen. ${ }^{54}$ Zur Wahrung der Rechtssicherheit stellte das BVerfG strenge Anforderungen an eine erfolgreiche Inhaltskontrolle:

»Schon aus Gründen der Rechtssicherheit darf ein Vertrag nicht bei jeder Störung des Verhandlungsgleichgewichts nachträglich in Frage gestellt oder korrigiert werden.

Handelt es sich jedoch um eine

- typisierbare Fallgestaltung, die eine

- strukturelle Unterlegenheit des einen Vertragsteils erkennen lässt, und sind die

- Folgen des Vertrages für den unterlegenen Vertragsteil ungewöhnlich belastend, ${ }^{55}$ so muss die Zivilrechtsordnung hierauf reagieren und Korrekturen ermöglichen. ${ }^{56}$

Dabei ist insbesondere von Bedeutung wie der Vertrag zustande kam.

Die verwendeten Begriffe definiert das BVerfG leider nicht. Der Begriff der strukturellen Unterlegenheit ist vertragsbezogen $\mathrm{zu}$ sehen und bedeutet, dass ein Vertragspartner in bestimmten Vertragssituationen typischerweise seine Interessen nicht durchsetzen kann, ${ }^{57}$ seine Selbstbestimmung im Sinne freier Willensentscheidung also regelmäßig eingeschränkt ist und zur Fremdbestimmung wird. ${ }^{58}$

Damit soll eine Missbrauchsabgrenzung getroffen werden, die nur einen Randbereich von evident einseitiger Belastung der Inhaltskontrolle unterzieht, denn im Bereich der Rechtsgeschäfte muss die richterliche Inhaltskontrolle - auch unter dem Aspekt des Vorranges des Gesetzes - Ausnahme bleiben.

Nach Forderungen in der Literatur, ${ }^{59}$ die sich als Antwort auf zwei grundlegende Entscheidungen des $\mathrm{BGH}^{60}$ zur völligen Ehevertragsfreiheit verstanden, hat das BVerfG auch für die Eheverträge die Inhaltskontrolle begründet. ${ }^{61}$ Es nimmt in veränderter Wortwahl folgende Voraussetzungen für die Korrektur durch Inhaltskontrolle an:

Am Schluss seiner Entscheidung hebt das BVerfG die vorhergehende Gerichtsentscheidung auf und verlangt Prüfung, ob diesen Prinzipien unter Heranziehung des geltenden Rechts Rechnung getragen werden kann. Anderenfalls regt es Verfahrensaussetzung bis zu einer Abhilfe durch den Gesetzgeber an.

53 BVerfGE 89, $214 \mathrm{ff}$. = NJW 1994, $36 \mathrm{f}$.

54 Später Bestätigung durch Kammerbeschlüsse: BVerfG, NJW 1994, 2749 f. und NJW 1996, 2021.

55 Eingriffsschwelle zivilrechtlicher Prüfung.

56 BVerfG, NJW 1994, 36, 38.

57 Vgl. Drexl, Die wirtschaftliche Selbstbestimmung des Verbrauchers, 1998, 276: situationsgebundene Vertragsschwäche.

58 BVerfGE 81, 242, 255.

59 Schwenzer, AcP 196 (1996), 88 ff. - hiergegen Grziwotz, FamRZ 1997, 586, 589; Büttner, FamRZ 1997, 600; Büttner, FamRZ 1998, 1, 4.

60 BGH, FamRZ 1996, 1536 = NJW 1997, 126 (schwangere Ehefrau unterzeichnet Verzicht drei Tage vor der Hochzeit, die davon abhängig war) und BGH, FamRZ 1997, 156 = NJW 1997, 192 (Ehemann unterließ Scheidung, weil Ehefrau Verzicht unterzeichnete).

61 BVerfGE 103, 89 ff. = DNotZ 2001, $222=$ MittBayNot 2001, $207=$ FamRZ 2001, 343 und BVerfG, DNotZ 2001, 708 = MittBayNot 2001, 485 = FamRZ 2001, 985. 
- besonders einseitige Aufbürdung vertraglicher Lasten (das dürfte der ungewöhnlichen Belastung in der Bürgschaftsentscheidung entsprechen) und

- erheblich ungleiche Verhandlungsposition.

Auffällig ist hierbei, dass nicht mehr von »struktureller Unterlegenheit « die Rede ist. Allerdings wird später bei der konkreten Fallprüfung ausgeführt, eine Situation der Unterlegenheit liege »regelmäßig« vor, wenn eine schwangere Frau sich vor die Alternative gestellt sehe, mit belastendem Ehevertrag zu heiraten oder ihr Kind unverheiratet zur Welt $\mathrm{zu}$ bringen. Auch von einer typisierbaren Fallgestaltung spricht das BVerfG nicht mehr.

Daneben nimmt das Gericht folgende spezifisch ehebezogene Argumentation vor:

- Art. 6 Abs. 1 GG setzt eine gesetzliche Ausgestaltung der Ehe voraus, so dass ein Teil des Eherechts herkömmlich zwingendes Recht sei.

- Mit Blick auf Art. 3 Abs. 2 GG sei verfassungsrechtlich geschützt eine Ehe, in der Mann und Frau in gleichberechtigter Partnerschaft zueinander stehen. Eine Grenze, die den staatlichen Schutzauftrag auslöse, sei daher erreicht, wenn der Vertrag nicht mehr Ausdruck und Ergebnis gleichberechtigter Lebenspartnerschaft sei.

- Die Eheschließungsfreiheit stehe der Inhaltskontrolle nicht entgegen. Das Eheversprechen selbst ist nicht Kompensation auferlegter Belastungen.

- Nach Art. 6 Abs. 4 GG sei schließlich die schwangere Verlobte als Mutter und nach Art. 6 Abs. 2 GG das Kind geschützt. Werde dessen Wohl durch den Ehevertrag nachhaltig gefährdet, greife die Schutzpflicht des Staates gegenüber dem Kind ein; dieses müsse vor verantwortungsloser Ausübung des Elternrechts geschützt werden.

Die Entscheidung des BVerfG löste eine Lawine von Urteilen der Obergerichte aus, die sich nun der Inhaltskontrolle von Eheverträgen widmeten. ${ }^{62}$ Mit seiner Entscheidung vom 11.2.2004 ${ }^{63}$ setzte der BGH die Vorgabe des BVerfG wohl ausgewogen in das Zivilrecht um und gewann somit für die Frage der Wirksamkeit und Anwendbarkeit von Eheverträgen ein Stück gesichertes Terrain zurück. Damit wurde der verfassungsrechtlich gegebene Anstoß genuin zivilrechtlich bewältigt. ${ }^{64}$ Inzwischen liegen weitere Entscheidungen des BGH zur Inhaltskontrolle von Eheverträgen vor. ${ }^{65}$ Die Urteile des BGH sollen hier nicht ausführlich dargestellt sein, da dies bereits anderenorts $^{66}$ und vielstimmig ${ }^{67}$ geschehen ist.

Damit aber hat die Rechtsprechung erst einen Anfang gemacht, die Inhaltskontrolle von Eheverträgen zu entwickeln. Viele Probleme harren noch gerichtlicher Entschei-

62 Vgl. die Zusammenstellung bei Münch, Ehebezogene Rechtsgeschäfte, 2004, Rdz. 360 ff.

63 BGH, NJW 2004, 930 f. $=$ FamRZ 2004, 601 ff. $=$ DNotZ 2004, 550 ff. $=$ ZNotP 2004, 157 ff.

64 Hierzu Diederichsen, AcP 198 (1998), 171, 234 ff.

65 Vom 6.10.2004 (AZ XII ZB 57/03), NJW 2005, 139 und (XII ZB 110/99), NJW 2005, 137; hierzu C. Münch, FamRZ 2005, 570 ff.; vom 12.1.05 (XII ZR 238/03) und je vom 25.5.05 (XII ZR 221/02 und XII ZR 296/01), die das Grundkonzept fortentwickeln. Danach ist die Teilnichtigkeit eines Ehevertrags möglich. Unterhaltsobergrenzen werden auch im Kernbereich anerkannt, wenn sie geeignet sind, ehebedingte Nachteile auszugleichen.

66 C. Münch, ZNotP 2004, $122 \mathrm{ff}$.

67 Bergschneider, FamRZ 2004, 1757 ff.; Borth, FamRZ 2004, 609 ff.; Brandt, MittBayNot 2004, 278 f.; Gageik, RNotZ 2004, 295 ff.; Grziwotz, FamRB 2004, 199, 239 ff.; Haußleiter/ 
dung. Für den Vertragsgestalter bestehen somit zahlreiche Rechtsunsicherheiten. Daher lohnt es, über die Besonderheiten familienrechtlicher Inhaltskontrolle nachzudenken, und ein Fundament zu schaffen, von dem aus die Einzelfragen bewältigt werden können. Dies soll in den nachfolgenden Thesen geschehen.

\section{Besonderheiten der Inhaltskontrolle von Eheverträgen}

\section{Missbrauchsabgrenzung - nicht Leitbildabweichung}

a) Leitbildmangel des dispositiven Familienrechts

Um im Rahmen der Inhaltskontrolle die besonders einseitige Aufbürdung vertraglicher Lasten feststellen zu können, müsste zunächst ein Maßstab entwickelt werden. Anhand dieses Maßstabes ist dann die Abweichung zu quantifizieren. ${ }^{68}$ Insoweit ist fraglich, ob das dispositive Eherecht des BGB zum Maßstab taugt.

Ob Dispositivnormen generell als Maßstab tauglich sind, lässt sich nicht einheitlich beantworten, denn Dispositivnormen können verschiedene Funktion einnehmen. Sie können reine Zweckmäßigkeitsregeln ohne vertieften Wertgehalt sein, sie können nur eine von den Parteien nicht getroffene Regelung gesetzlich vorsehen und insoweit den Parteien eigene Abreden ersparen ${ }^{69}$ oder sie können ein Gerechtigkeitsgebot enthalten. ${ }^{70}$ Sofern sie ein Gerechtigkeitsgebot enthalten, können sie zum Leitbild erhoben werden, so wie dies im Rahmen des AGB-Rechts geschieht. ${ }^{71}$

Bei den Dispositivnormen des Eherechts herrscht jedoch die Meinung vor, dass diesen Normen gerade kein Leitbildcharakter ${ }^{72}$ zukommt. So verabschiedet sich das bürgerlich-rechtliche Ehebild nach dem 1. Eherechtsreformgesetz vom Institutionendenken. ${ }^{73}$ Es herrscht ein interindividuelles oder personal-individualistisches Eheverständnis vor, das nur noch wenige Grundstrukturen vorgibt und den Ehegatten ansonsten Freiheit der Rollenverteilung gewährt. ${ }^{74}$ Angesichts der verschiedenen Ehe-

Schiebel, NJW Spezial 2004, 7; Klam, INF 2004, 315 f.; Koch, NotBZ 2004, 147 f.; Kornexl, FamRZ 2004, 1609 f.; Langenfeld, ZEV 2004, 311 ff.; J. Mayer, FPR 2004, 363 ff.; C. Münch, ZNotP 2004, 122 ff.; Rakete-Dombek, NJW 2004, 1273 ff.; Rauscher, DNotZ 2004, 524 ff.; Wachter, ZFE 2004, 132 ff.

$68 \mathrm{Zu}$ dieser allgemeinen Methodik bei der Inhaltskontrolle: Fastrich, (FN 4), $224 \mathrm{ff}$.

69 Medicus, AcP 192 (1992), 35, 47.

70 Vgl. hierzu etwa BGHZ 41, 151, 154; Ennecerus/Nipperdey, Allgemeiner Teil des Bürgerlichen Rechts, 15. Aufl., 1. Halbband, 1959, § 49 III, S. 301; Krafka, DNotZ 2002, 677, 686; Mikat, Festschrift für Wilhelm Felgentraeger, 1969, 323, 333; Weick, NJW 1978, 11 ff. auch mit historischen Bezügen. Zu allgemein und ohne Differenzierung zwischen verschiedenen dispositiven Normen: Becker, WM 1999, 709, 717.

71 BGHZ 41, 151, 154; zur Entwicklung des Leitbildgedankens: Krafka, DNotZ 2002, 677, 686; Weick, NJW 1978, $11 \mathrm{ff}$.

72 Eine Zusammenstellung von Eheleitbildern, Ehemotiven und Eheverträgen im Spiegel der Literatur: geben Dauner-Lieb/Sanders, Festschrift für Ingrid Groß, 2004, 39 ff.

73 Gernhuber, FamRZ 1979, 193, 195.

74 Badura in Maunz/Dürig/Herzog, Grundgesetz Kommentar, (Stand 8/2000), Art. 6, Rdz. 27; Hepting, Ehevereinbarungen, 1984, 28; Reinhart, JZ 1983, 184, 185; Hattenhauer, FamRZ 1989, 225 ff. Vgl. nur zur Begründung des Gesetzentwurfes den Bericht des Rechtsausschusses, BT-Drs. 7/4361, 8. 
typen $^{75}$ bzw. Ehekonstellationen ${ }^{76}$ benötigen die Ehegatten den gesetzlich belassenen Gestaltungsspielraum, um die rechtlichen Folgen ihrer Ehe der gelebten Ehekonstellation anzupassen. Das BGB hingegen gibt Orientierungshilfe nur für die Alleinverdiener- oder Hausfrauenehe. Für andere Ehetypen fehlt es dagegen völlig an einem Leitbild in Form dispositiver Vorschriften. ${ }^{77}$ So kommt es, dass das geltende Eherecht als eigentümlich zerrissen empfunden wird, weil es einerseits der Autonomie der Ehegatten vertraut, »andererseits aber Normkomplexe mit allgemeinem Geltungsanspruch bewahrt, die aus der Anschauung eines einzigen Ehetyps konzipiert wurden. ${ }^{78}$ Aus diesem Grunde ergeht auch der Ratschlag, dass die Ehegatten ihr »privates Eheleitbild « vertraglich ordnen, also auch personale Elemente in den Ehevertrag aufnehmen. ${ }^{79}$ Ein Rat, der an Aktualität gewonnen hat, seit der BGH Konsequenzen für die Inhaltskontrolle von Eheverträgen auch vom gewählten Ehemodell abhängig macht.

Somit kann das dispositive Eherecht nicht als Leitbild angesehen werden, das zum einheitlichen Maßstab aller Eheverträge taugt. ${ }^{80}$ Vielmehr wird in $\S \S 1353,1356$ BGB geradezu das grundrechtlich geschützte Recht der Ehegatten verbürgt, ihre eheliche Lebensgemeinschaft eigenverantwortlich und frei von gesetzlichen Vorgaben entsprechend ihren individuellen Vorstellungen und Bedürfnissen zu gestalten. ${ }^{81}$ Dies muss dann auch für die Scheidungsfolgen gelten.

\section{b) Grundgesetzliches Leitbild?}

Weiter ist zu fragen, ob sich aus Art. 6 Abs. 1 GG ein verfassungsrechtliches Leitbild für die verschiedenen Ehetypen ergibt. Hierbei setzt die Bestimmung der Ehe im Sinne des Art. 6 Abs. 1 GG allerdings wiederum den Blick auf deren einfachgesetzliche Ausgestaltung voraus, ${ }^{82}$ denn Art. 6 Abs. 1 GG geht von der vorgefundenen Lebensform der bürgerlich-rechtlichen Ehe aus. ${ }^{83}$

75 Vgl. Gernhuber, Eherecht und Ehetypen, 1981; Langenfeld, Sonderheft DNotZ 1985, 167, 170 f.; Langenfeld, FamRZ 1987, 9 ff.

76 Vgl. C. Münch, Ehebezogene Rechtsgeschäfte, 2004, Rdz. 2404 ff.

77 Coester-Waltjen, 50 Jahre BGH, Festgabe aus der Wissenschaft, Band 1, 2000, 985, 986; BGH v. 25.5.05 (XII ZR 296/01).

78 Gernhuber, FamRZ 1979, 193, 195.

79 Hattenhauer, FamRZ 1989, 225, 232.

80 Grziwotz, FamRB 2004, 199, 202, 239, 240; ders., DNotZ-Sonderheft 1998, 228, 249; Hahne, DNotZ 2004, 84, 88; Palandt/Brudermüller, BGB, 64. Aufl., 2005, § 1360, Rdz. 8; Schwenzer, AcP 196 (1996), 88, 112.

81 BGH, NJW 2004, 930 f. = FamRZ 2004, 601 ff. = ZNotP 2004, 157 ff. = DNotZ 2004, 550 ff., Ziffer III, 1a) der Gründe; Gernhuber, FamRZ 1979, 193, 201 spricht vom Polymorphismus in der Ehe. BGH v. 25.5.05 (XII ZR 296/01) gibt darüber hinaus auch für die Alleinverdienerehe das Recht zu abweichender Gestaltung.

82 Gröschner in Dreier, Grundgesetz Kommentar, 2. Aufl., 2004, Art. 6, Rdz. 36; Loschelder, FamRZ 1988, 333, 334; Gellermann, Grundrechte im einfachgesetzlichen Gewande, 2000, 127 ff.; Pieroth/Kingreen, KritV 2002, 219, $220 \mathrm{f}$.

83 BVerfGE 31, 58, 69; Ruffert, Vorrang der Verfassung und Eigenständigkeit des Zivilrechts, 2001, 424: Erkenntnisvorrang des Zivilrechts schlägt auf die verfassungsrechtliche Begriffsbildung durch. 
Art. 6 Abs. 1 GG bezieht auch die gescheiterte Ehe noch in seinen Schutzbereich ein, da die Ehe auf Dauer angelegt ist, ${ }^{84}$ und der Gesetzgeber daher gehalten ist, der fortwirkenden personalen Verantwortung der Ehegatten füreinander bei Regelung der Scheidungsfolgen Rechnung zu tragen. ${ }^{85}$ Allerdings hat er hierbei einen ganz erheblichen Gestaltungsspielraum. ${ }^{86}$

Der Ehebegriff des Art. 6 Abs. 1 GG ist frei von inhaltlichen Vorgaben. Der Verfassung liegt danach kein bestimmtes Ehemodell zugrunde. ${ }^{87}$ Allerdings wird von der Prägung durch ein partnerschaftliches Ehemodell gesprochen, das aus der rechtlichen Durchsetzung der Gleichberechtigung resultiert. ${ }^{88}$ Danach ist eine solche Ehe geschützt, in der Mann und Frau in gleichberechtigter Partnerschaft zueinander stehen. ${ }^{89}$ Ansonsten werden nur die Grundsätze der Einehe, der Geschlechtsverschiedenheit, der zeitlichen Unbeschränktheit der Lebensgemeinschaft ${ }^{90}$ sowie die Verpflichtung zur ehelichen Lebensgemeinschaft ${ }^{91}$ als Strukturprinzipien genannt, die der Disposition des einfachen Gesetzgebers entzogen sind, solange kein verfassungsrechtlich bedeutsamer Wandel des Eheverständnisses stattgefunden hat. ${ }^{92}$

Auffällig ist, dass es schwer fällt, den geschützten Bereich der Ehe und Familie präzise zu bestimmen. Auch die einfachgesetzlichen Regelungen enthalten detaillierte Vorgaben für das Scheitern der Ehe und die Folgen, setzen aber den positiven Gehalt der Ehe stillschweigend voraus. ${ }^{93}$ Daraus wird gefolgert, Art. 6 Abs. 1 GG halte eine Sphäre gerade von staatlicher Durchdringung frei, in der die Ehegatten ihre Lebensform in eigener Entscheidung entwickeln können. ${ }^{94}$ Art. 6 Abs. 1 GG schützt somit die Gestaltungsfreiheit der Ehegatten. Aufgrund des Abwehrcharakters muss ihnen diese erhalten bleiben, so dass grundsätzlich auch die Wahl verschiedener Ehetypen ohne Bindung an überkommene Rollenverteilungen ${ }^{95}$ und der Abschluss entsprechender Eheverträge durch Art. 6 Abs. 1 GG geschützt sind. ${ }^{96}$ Diskutiert werden sogar ehe-personalrechtliche Ehetypen erschwerter oder erleichterter Scheidungen oder die gesetzliche Zulassung rechtsindifferenter Ehen. ${ }^{97}$

84 Badura in Maunz/Dürig/Herzog, (FN 74), Art. 6, Rdz. 45.

85 BVerfGE 53, 224, 250; Gröschner in Dreier, (FN 82), Art. 6, Rdz. 62.

86 BVerfGE 53, 224, 245.

87 Burgi in Berliner Kommentar zum Grundgesetz, 2003, Art. 6, Rdz. 18.

88 Coester-Waltjen in von Münch/Kunig, Grundgesetz, 5. Aufl., 2000, Art. 6, Rdz. 4.

89 BVerfG, DNotZ 2001, 222 ff. = FamRZ 2001, 343 ff.

90 Badura in Maunz/Dürig/Herzog, (FN 74), Art. 6, Rdz. 45; Coester-Waltjen, (FN 88), Art. 6, Rdz. 8 ff.

91 Hattenhauer, FamRZ 1989, 225, 231.

92 Coester-Waltjen, (FN 88), Art. 6, Rdz. 13.

93 Loschelder, FamRZ 1988, 333, 336.

94 Loschelder, FamRZ 1988, 333, 336; Friauf, NJW 1986, 2595, 2598: »binnenfamiliäre Organisationsautonomie«.

95 BVerfGE 87, 234, 258: Art. 3 Abs. 2 GG verbiete die Festschreibung überkommener Rollenverteilungen.

96 Rauscher, FamRZ 1997, 1121, 1123; Coester-Waltjen, (FN 88), Art. 6, Rdz. 27.

97 Vgl. hierzu Bosch in Symposium Beitzke, 1989, 9 ff.; Herzog, Bitburger Gespräche Jahrbuch 1988, 15 ff.; Hattenhauer, ZRP 1985, 200 ff. 
Somit ist der Ehebegriff des Art. 6 Abs. 1 GG frei von für alle geltenden inhaltlichen Vorgaben mit Ausnahme weniger Strukturprinzipien.

c) Grundsatz der Ehevertragsfreiheit - gesetzliche Regelung ehelicher Verträge

Beachtlich ist ferner, dass das Familienrecht ganz detaillierte Vorschriften dazu enthält, welche vertraglichen Regelungsmöglichkeiten die Ehegatten beim Ehevertrag haben, welche Rechte unverzichtbar sind und welche Form für den Vertrag einzuhalten ist. ${ }^{98}$ So lässt $§ 1408$ BGB ausdrücklich zu, dass die Ehegatten ihren Güterstand und den Versorgungsausgleich durch notariell beurkundeten Ehevertrag ( $\$ 1410$ BGB) regeln. $§ 1587$ o Abs. 2 BGB ordnet für scheidungsbezogene Vereinbarungen des Versorgungsausgleichs eine richterliche Inhaltskontrolle an. §1378 Abs. 3 BGB regelt Rechtsgeschäfte über die konkrete Zugewinnausgleichsforderung vor deren Entstehen. $\S 1585$ c BGB schließlich erlaubt ausdrücklich, dass die Ehegatten formfrei Vereinbarungen über den nachehelichen Unterhalt treffen. Dies steht im Gegensatz zu den ansonsten zwingend ausgestalteten Unterhaltstatbeständen der $\S \S 1360 \mathrm{a}$ Abs. 3, 1361 Abs. 4, 1614 Abs. 1 und 16151 Abs. 3 Satz 1 BGB.

Ein Gericht, das im Sinne einer strikten Halbteilung schon jede Abweichung von den gesetzlichen Scheidungsfolgen als einseitige Belastung einstuft, ${ }^{99}$ hätte eigentlich vorrangig diese familienrechtlichen Vorschriften, welche die Vertragsfreiheit ausdrücklich gewähren, auf ihre Verfassungsmäßigkeit prüfen müssen. Sie müssten dann verworfen werden, wenn sie das gebotene Maß an Schutz nicht leisten können, ${ }^{100}$ entweder weil sie zu viel an Wahlfreiheit geben oder weil sie - wie etwa $§ 1585$ c BGB keine gesonderte Form vorschreiben und so auf einen möglichen Übereilungsschutz und Rechtsbelehrung verzichten. Der BGH jedoch stellt den Grundsatz an den Beginn seiner Ausführungen, dass die gesetzlichen Regelungen über die Scheidungsfolgen grundsätzlich der vertraglichen Disposition der Ehegatten unterliegen, es also keinen unverzichtbaren Mindestgehalt an Scheidungsfolgen gibt. ${ }^{101}$ Die Ehevertragsfreiheit wird damit grundsätzlich anerkannt. Grenze ihrer Ausübung ist nicht die Abweichung von dispositiven Normen, sondern nur der Missbrauch der gegebenen Ehevertragsfreiheit durch Verletzung des Schutzzweckes der gesetzlichen Regelung, d.h. durch Verstoß gegen die Grundprinzipien des Art. 6 Abs. 1 GG.

\section{d) Ehekonstellation für Inhaltskontrolle entscheidend}

Das BVerfG stellt in der Ehevertragsentscheidung zwei Strukturprinzipien heraus, die es als Leitbild für die Frage ansehen möchte, ob eine einseitige Lastenverteilung vorliegt. Dies ist zum einen das aus Art. 6 Abs. 1 i.V.m. Art. 3 Abs. 2 GG folgende Leitbild gleichberechtigter Partnerschaft und zum anderen das Leitbild unterhaltsrechtli-

98 Hierzu Röthel, NJW 2001, 1334.

99 So etwa das OLG München, FamRZ 2003, 35 mit Anm. Bergschneider = MittBayNot 2003, 136 mit Anm. Münch (107 ff.)

100 Vgl. zur Verfassungswidrigkeit des § 1408 BGB: Dauner-Lieb, FF 2002, 151, 153.

101 BGH, NJW 2004, 930 f. = FamRZ 2004, 601 ff. = ZNotP 2004, 157 ff. = DNotZ 2004, 550 ff., Ziffer III, 1 - vor a) der Gründe. 
cher Verantwortung aufgrund Elternschaft. ${ }^{102}$ Diese beiden Strukturprinzipien sollen ungeachtet des gewählten Ehemodells eine gewisse Grundprägung der Ehe und - bei Kindern - der Familie bilden, von der sich die Ehegatten auch angesichts ihrer Gestaltungsfreiheit nicht vollständig entsolidarisieren können. Dies entspricht der Aussage des $\S 1353$ Abs. 1 Satz 2 BGB, dass die Ehegatten füreinander Verantwortung tragen. Auf dieser Grundannahme beruht letztlich die Ehevertragsentscheidung des BVerfG. An anderer Stelle hat das BVerfG aber zugleich betont, das Wesen der Ehe sei auch durch die Selbstverantwortlichkeit der Ehegatten gekennzeichnet, welche dem Schutzauftrag des Staates Grenzen setze. ${ }^{103}$

Weder aus der dispositiven gesetzlichen Regelung noch aus dem Schutzbereich des Art. 6 Abs. 1 GG ergibt sich somit, dass schon jede Abweichung von den dispositiven Scheidungsfolgen als Aufbürdung vertraglicher Lasten gesehen werden kann. Auch die gegenseitige Verantwortung macht das Eherecht nicht zwingend, ${ }^{104}$ sondern sie gibt nur eine Grundsolidarität vor, ohne dass diese sich in zwingender Halbteilung ausdrücken müsste.

Maßstab für die Inhaltskontrolle von Eheverträgen müssen also demnach sein:

(1) die vom BVerfG hervorgehobenen Grundprinzipien gleichberechtigter Partnerschaft und unterhaltsrechtlicher Verantwortung aufgrund Elternschaft,

(2) das unter Respektierung dieser Grundlage von den Ehegatten gewählte Ehemodell.

Regelungen eines Ehevertrages, die dieser Ehekonstellation entsprechen, sind im Rahmen einer Inhaltskontrolle nicht zu beanstanden. Der BGH hat diese Grundprinzipien des BVerfG mit seiner Kernbereichslehre in das Zivilrecht hinein entwickelt zu einer Stufenfolge, die allerdings nicht starr ist, sondern die Schutzwürdigkeit des Ehegatten und die Disponibilität abhängig vom gewählten Ehemodell sein lässt.

Die richtige Fragestellung muss sich also darauf konzentrieren, ob die getroffenen Regelungen der gewählten Ehekonstellation entsprechen. ${ }^{105}$ Dabei darf diese Ehekonstellation nicht zu grobmaschig gesehen werden. So ist zwar die Einverdienerehe ein Ehetyp, aber dieser lässt sich erneut untergliedern, so dass es in der »Diskrepanzehe « andere ehetypgerechte Regelungen geben kann als in der »Normalverdienerehe $« .{ }^{106}$ Zudem sind verschiedene Ehephasen zu unterscheiden, so dass besser von einer Ehekonstellation die Rede ist. ${ }^{107}$ Hier wird mit Fallgruppenbildung ein Maßstab zu entwickeln sein, an dem sich die Abweichungen messen lassen.

Die völlig gleiche Rechtsfolge des Ehevertrages - etwa: jeder Vertragsteil verzichtet auf Unterhalt - darf daher nicht als formal gleichwertig angesehen werden, sondern

102 So Heidrich/Heins, NotBZ 2001, 140, 143.

103 BVerfGE 31, 58, 84.

104 Grziwotz, FamRB 2004, 199, 200.

105 Vgl. Dauner-Lieb, FF 2004, 65, 66; ausführlich Dauner-Lieb, Brennpunkte des Familienrechts 2003, $1 \mathrm{ff}$.

106 Insoweit ist eine starre Halbteilung nicht geboten. A.A. wohl Dauner-Lieb, FF 2004, 65, $66 \mathrm{f}$.

107 Kritisch zum festen Ehetyp Kornexl, FamRZ 2004, 1609 f. 
kann für jeden Ehegatten je nach der ehelichen Rollenverteilung völlig unterschiedliche Auswirkungen haben. ${ }^{108}$

Die Inhaltskontrolle von Eheverträgen ist somit angesichts der Offenheit des Eherechts für individuelle Gestaltung und angesichts des dargelegten Gewichts der Privatautonomie eine Missbrauchskontrolle und keine Leitbildabweichungskontrolle. ${ }^{109}$

\section{Typisierte Fallgestaltung struktureller Unterlegenheit}

a) Übertragung der Anforderungen der Bürgschaftsentscheidung

Das BVerfG hat bei seinen Entscheidungen zur Inhaltskontrolle von Eheverträgen einerseits auf die Bürgschaftsentscheidung Bezug genommen. Andererseits hat das BVerfG nicht mehr die Voraussetzungen der typisierten Fallgestaltung und der strukturellen Unterlegenheit im Wortlaut wiederholt, sondern eine erheblich ungleiche Verhandlungsposition gefordert und später ausgeführt, diese liege »regelmäßig « vor, wenn eine schwangere Frau sich vor die Alternative gestellt sehe, mit belastendem Ehevertrag zu heiraten oder ihr Kind unverheiratet zur Welt zu bringen. Aus der Wortwahl »regelmäßig « lässt sich schließen, dass auch hier mit dem Argument der Rechtssicherheit eine typische Fallsituation und nicht eine Einzelfallprüfung gefordert wird. Zudem beinhaltet die Vertragsfreiheit auch die Pflicht des Gesetzgebers, rechtsgeschäftliche Gestaltungsmittel zur Verfügung zu stellen, die als rechtsverbindlich zu behandeln sind und auch im Streitfall durchsetzbare Rechtspositionen begründen. ${ }^{110}$ Das bedeutet, dass das Instrument des Ehevertrages nicht vollständig ausgehöhlt werden darf. Solches würde aber geschehen, wenn ohne Feststellung einer Situation der Unterlegenheit jeder Ehevertrag der Inhaltskontrolle unterläge.

Zudem betont gerade das BVerfG im Zusammenhang mit Art. 6 Abs. 1 GG, dass die Ehe durch die Selbstverantwortlichkeit der Ehegatten gekennzeichnet sei und dies dem Schutzauftrag des Staates Grenzen setze. ${ }^{11}$ Dieser Gedanke, den das BVerfG zur Eheschließung entwickelte, lässt sich auf die vermögensrechtliche Ausgestaltung der Ehe übertragen. In diesem Zusammenhang bedeutet Freiheit eben auch Freiheit zur Unvernunft, ${ }^{112}$ wenn die Entscheidung selbstverantwortlich fällt.

Somit erfordern also sowohl die grundrechtlich geschützte Privatautonomie wie die Rechtssicherheit und Art. 6 Abs. 1 GG selbst, dass nicht jeder Ehevertrag der Inhaltskontrolle unterliegt, sondern nur solche Eheverträge, die durch eine Ungleichgewichtslage gekennzeichnet sind. Wer danach in freier Entscheidung ein Risiko sieht und dieses eingeht, der muss sich am Vertrag festhalten lassen. ${ }^{113}$

108 BVerfGE, DNotZ 2001, 222 = FamRZ 2001, 343.

109 Hier liegt das Missverständnis, auf dem das Urteil des OLG München (FamRZ 2003, 36 mit Anm. Bergschneider) als Vorinstanz zum BGH-Urteil vom 11.2.2004 beruht. Vgl. schon C. Münch, MittBayNot 2003, 107 ff. Daher konnte man aus der Sicht der Entscheidung des BVerfG eine solche radikale Einschränkung der Vertragsfreiheit auch nicht erwarten (so Rakete-Dombek, NJW 2004, 1273, 1274).

110 BVerfG, NJW 1994, 36, 38.

111 BVerfGE 31, 58, 84.

112 Coester-Waltjen, (FN 77), 985, 1002. 
b) Vertragsfreiheit im Näheverhältnis

Im Unterschied zu den bisher beim Thema Inhaltskontrolle in der Regel behandelten Austauschverträgen des Wirtschaftsrechts geht es nun um den Gestaltungsspielraum in höchstpersönlichen Beziehungen, ${ }^{114}$ die zudem die gesamte Existenz umfassen können. ${ }^{115}$ So bricht sich das rationale Kalkül, wo Nähe herrscht. ${ }^{116}$ Man spricht auch von unverträglichen Handlungslogiken familienrechtlicher Solidarität und wirtschaftlicher Rationalität. ${ }^{117}$ Zwar wurde auch bei der Bürgschaftsentscheidung auf das besondere familienrechtliche Verhältnis zwischen Hauptschuldner und Bürge abgestellt und daraus auf eine emotionale Entscheidung anstelle einer abwägenden rationalen geschlossen. Andererseits stehen sich die Ehegatten als Vertragspartner mit je eigenen Interessen gegenüber. Anders als im Bürgschaftsfalle, wo ein Familienangehöriger dem anderen gegen die Bank hilft und auf seiner Seite steht, besteht beim Ehevertrag die typische Vertragssituation des Aushandelns eigener Rechtsstandpunkte zweier sich gegenüberstehender Vertragsteile. ${ }^{118}$

Die persönliche Nähe kann somit in vielerlei Hinsicht Entscheidungen beeinflussen. Sie ist keineswegs allein schon Indiz für ein stets vorliegendes Verhandlungsungleichgewicht.

\section{c) Formvorschriften zur Stärkung der Selbstverantwortlichkeit}

Zur Stärkung dieser Selbstverantwortlichkeit hat das Gesetz in vielen, nicht aber in allen Fällen besondere Formvorschriften für den Abschluss von ehevertraglichen Abreden aufgestellt. So ist in $\S 1410$ BGB für die güterrechtlichen Regelungen und ehevertragliche Versorgungsausgleichsregelungen und in $\S 1587$ o Abs. 2 Satz 1 BGB für scheidungsbezogene Versorgungsausgleichsregelungen die notarielle Beurkundung angeordnet. Unterhaltsverzichte sind dagegen nach $\S 1585 \mathrm{c}$ BGB formlos möglich, obwohl der Unterhalt gerade nach der neuen Rechtsprechung des BGH den am wenigsten disponiblen Kernbereich der Scheidungsfolgen ausmacht. Dies wurde schon lange aus berufenem Munde als verfehlt kritisiert. ${ }^{119}$ Neuerdings schlägt sogar die unterhaltsrechtliche Literatur vor, trotz des Fehlens eines Beurkundungszwangs für Unterhaltsverzichte die notarielle Form zu wahren, da dies aufgrund der neutralen Urkundsperson und des einzuhaltenden Verfahrens den Verzicht sicherer mache. ${ }^{120}$ Der

113 Vgl. etwa Hillgruber, AcP 191 (1991), 69, 85; Langenfeld, ZEV 2004, 311, 314.

114 Hohmann-Dennhardt, 1. Jahresarbeitstagung des Notariats, 2003, 6.

115 Coester-Waltjen, (FN 77), 985, 986.

116 Hohmann-Dennhardt, (FN 114), 14; auf die Besonderheiten der familienrechtlichen Situation verweist auch Dauner-Lieb (FN 105), 1, 18.

117 Teubner, KritV 2000, 388 ff., zu der Bürgschaftsentscheidung.

118 So vertritt auch Coester-Waltjen, (FN 77), 985, 1001 ff., dass die Richtigkeitsgewähr trotz des familiären Näheverhältnisses eingreife.

119 Bosch, Festschrift für Habscheid, 1989, 23, 42; Langenfeld, DNotZ 1985, 167, 173. Dazu, dass bei Übernahme des $\S 72$ EheG der Anwendungsbereich vorsorgender Eheverträge völlig falsch eingeschätzt wurde: Schreiber, Gestaltungsfreiheit in Eheverträgen in rechtsdogmatischer und rechtstatsächlicher Sicht, 1983, $73 \mathrm{f}$.

120 Wendl/Staudigl/Pauling, Das Unterhaltsrecht in der familienrichterlichen Praxis, 6. Aufl., 2004, § 6, Rdz. 609; Bergschneider, FamRZ 2001, 1337, 1338. 
15. Deutsche Familiengerichtstag empfiehlt daher die Einführung einer entsprechenden Formvorschrift. ${ }^{121}$ Ohne dass dies hier im Detail nachgezeichnet werden kann, ${ }^{122}$ vermag die notarielle Beurkundung viele Ungleichgewichtslagen zu beseitigen. Grenzen allerdings sind dem bei seelischen Zwangslagen und evidenter Benachteiligung des Kindeswohls gesetzt.

\section{d) Zwischenergebnis}

Nicht jeder Ehevertrag und auch nicht jeder Ehevertrag, bei dem Verzichte auf Scheidungsfolgen erklärt werden, ist daher Ausdruck einer ungleichen Verhandlungsposition. Nur wenn eine solche Unterlegenheit mindestens typischerweise vorliegt, kann ein Ehevertrag der richterlichen Inhaltskontrolle unterfallen.

\section{Subjektive Voraussetzungen - Kontrollinstrumentarium}

Haben wir die ehevertragliche Inhaltskontrolle als Missbrauchskontrolle kennengelernt, die bei typischer Unterlegenheit greift, so ist nun noch nach den subjektiven Voraussetzungen im Rahmen der Inhaltskontrolle zu fragen. Die Empfehlungen des 15. Deutschen Familiengerichtstages etwa sehen vor, dass die subjektiven Kriterien (Dominanz eines Vertragspartners unter Ausnutzung einer ungleichen Verhandlungsposition) Voraussetzung für die Inhaltskontrolle sein sollen. Bei einem Globalverzicht soll das Vorliegen dieser Voraussetzungen widerlegbar vermutet werden. ${ }^{123}$ Andererseits wird geraten, die krampfhafte Suche nach einer Ungleichgewichtslage zu beenden, da sie am Kern des Problems vorbeigehe. ${ }^{124}$

Bilden wir das Beispiel der schwangeren Verlobten, die ihrem Verlobten aber von der Schwangerschaft nichts gesagt hat. Wird in dieser Situation ein Ehevertrag geschlossen, so sieht sich die schwangere Verlobte zwar einer Zwangslage ausgesetzt. Der Vertragspartner kennt diese aber nicht einmal und kann sie daher auch nicht ausnutzen. Soll der Ehevertrag hier auch ohne ein Ausnutzen der Imparität seitens des Verlobten nichtig sein?

Die Frage nach den subjektiven Kriterien lässt sich nicht isoliert betrachten von der Frage, mit welchem Kontrollinstrumentarium die Inhaltskontrolle durchgeführt werden soll. Die Auswahl des hierfür geeigneten Instrumentariums hat das BVerfG den Zivilgerichten überlassen.

Der BGH hat dies so umgesetzt, dass er einerseits eine Wirksamkeitskontrolle nach $\S 138$ BGB durchführt und andererseits - vor allem, aber eben nicht nur - bei veränderten Umständen eine Ausübungskontrolle nach $\S 242$ BGB. ${ }^{125}$ Diese Ausübungs-

121 C.V., FuR 2004, 18, 22. So nunmehr auch der Referentenentwurf des BMJ, FamRZ 2005, 1041, 1043. Auch im Hinblick auf die Bürgschaftsfälle wurde schon geraten, § 766 BGB durch den Zwang zur notariellen Beurkundung zu verstärken, Floren, Grundrechtsdogmatik im Vertragsrecht, 1999, 419.

122 Ausführlich C. Münch, DNotZ 2004, $901 \mathrm{ff}$.

123 Empfehlungen A III.4., FuR 2004, 18, 20.

124 Dauner-Lieb, FF 2001, 129, 130.

125 BGH, NJW 2004, 930 f. = FamRZ 2004, 601 ff. = ZNotP 2004, 157 ff. = DNotZ 2004, $550 \mathrm{ff}$. 
kontrolle wird vielmehr auch als eine Auffangkontrolle durchgeführt werden, wenn die Voraussetzungen für eine Nichtigkeit des Vertrages aufgrund der durchgeführten Wirksamkeitskontrolle nicht vorliegen. ${ }^{126}$ Hierbei können sich durchaus jeweils verschiedene Anforderungen an die subjektiven Voraussetzungen ergeben.

\section{a) Wirksamkeitskontrolle}

Die vorhergehende Analyse der allgemeinen Inhaltskontrolle hat ergeben, dass für eine Inhaltskontrolle, die im Vorfeld des § 138 BGB angesiedelt ist, mindestens eine typisierbare Fallgestaltung struktureller Unterlegenheit im Sinne einer situativen Vertragsschwäche Voraussetzung ist. Damit ist man auch von der verkrampften Suche nach einer Ungleichgewichtslage ${ }^{127}$ im Einzelfall ein Stück befreit.

Um zur Nichtigkeit eines Ehevertrages nach $\S 138$ BGB zu gelangen, bedarf es jedoch nach hier vertretener Auffassung mehr. Hier müssen die allgemeinen Voraussetzungen des § 138 BGB gegeben sein, denn die Nichtigkeit eines Ehevertrages wegen Sittenwidrigkeit kann nur in Extremfällen in Betracht kommen. Der BGH spricht in diesem Zusammenhang von einem $»$ Verdikt $\ll,{ }^{128}$ also einer Verurteilung «. Schon dies deutet darauf hin, dass eine solche Sittenwidrigkeit nur nach den Voraussetzungen des $\S 138$ BGB vorliegen soll. Hierbei ist nicht von einer speziell familienrechtlichen Auslegung des $\S 138$ BGB auszugehen, ${ }^{129}$ wohl aber von einer grundrechtsgeleiteten Interpretation. ${ }^{130}$

Für eine Wirksamkeitskontrolle ist die Prüfung nach § 138 BGB gemäß den Regeln vorzunehmen, die sich zu $\S 138$ BGB herausgebildet haben. ${ }^{131}$ Hierbei ist die objektive Ungleichgewichtslage im Einzelfall zum Zeitpunkt des Vertragsabschlusses festzustellen, die einer Zwangslage im Sinne des § 138 BGB entspricht. Die Kriterien der Sittenwidrigkeit sind auch im Rahmen des $§ 138$ BGB nicht trennscharf abzugrenzen. ${ }^{132}$ Häufig ergibt sich die Sittenwidrigkeit erst aus der Summenwirkung verschiedener negativer Faktoren (sog. Sandhaufentheorem). ${ }^{133}$ Hier ist deshalb eine zusammenfassende Würdigung von Inhalt, Beweggrund und Zweck des Rechtsgeschäftes durchzuführen, ${ }^{134}$ so wie es der BGH für die Inhaltskontrolle von Eheverträgen vorsieht. Allerdings gibt es auch Fälle, wo das Vorliegen eines einzigen Grundes bereits die Sittenwidrigkeit nach sich zieht. Dies gilt insbesondere für die sog. Inhaltssitten-

126 Hierzu schon Münch, ZNotP 2004, 122, 128; vgl. nun BGH, NJW 2005, 139 f.

127 Dauner-Lieb, FF 2001, 129, 130 mit der Forderung, diese aufzugeben.

128 BGH, NJW 2004, 930 f. = FamRZ 2004, 601 ff. = ZNotP 2004, 157 ff. = DNotZ 2004, 550 ff., Ziffer III.3a) der Urteilsgründe.

129 So aber wohl Goebel, FamRZ 2003, 1513 ff.

130 BVerfGE 99, 185, 196; Hermes, VVDStRL 61, 119, 121; schon Schmidt-Salzer, NJW 1970, 8, 12 spricht von einer inhaltsprägenden Funktion der Grundrechte.

131 Vgl. Gageik, RNotZ 2004, 305 ff.

132 Zur Unschärfe des Typus-Begriffs: Münchener Kommentar/Mayer-Maly/Armbrüster, 4. Aufl., 2001, § 138, Rdz. 32.

133 Staudinger/Sack, BGB, Neubearbeitung 2003, § 138, Rdz. 57 f.; Münchener Kommentar, (FN 132), § 138, Rdz. 27.

134 BGHZ 107, 92, 97; BGHZ 86, 82, 88; Münchener Kommentar, (FN 132), § 138 Rdz. 30; Palandt/Heinrichs, (FN 80), § 138, Rdz. 8. 
widrigkeit. Ein Rechtsgeschäft, das gegen grundlegende Werte der Rechts- oder Sittenordnung verstößt, kann nicht anerkannt werden und ist daher unabhängig von weiteren Voraussetzungen und subjektiven Gegebenheiten nichtig. ${ }^{135}$

Lässt sich eine solche individuelle Ungleichgewichtslage nicht erweisen oder stellt sich im Rahmen der Prüfung eine Milderung der evident einseitigen Belastung durch Kompensationen heraus, so führt die Wirksamkeitskontrolle nicht zur Sittenwidrigkeit.

Schon im Rahmen der allgemeinen Dogmatik des $\S 138$ BGB ist nicht unumstritten, inwiefern subjektive Voraussetzungen für das Bejahen der Sittenwidrigkeit vorliegen müssen. Bei der geschilderten Inhaltssittenwidrigkeit wird solches nicht gefordert. Ansonsten geht wohl die noch herrschende Ansicht davon aus, dass neben den objektiven Elementen auch die Verwirklichung eines subjektiven Tatbestandes erforderlich ist. Danach ist zumindest die Kenntnis derjenigen Tatumstände erforderlich, welche die Sittenwidrigkeit begründen - ausreichend ist auch ein bewusstes Verschließen oder Entziehen vor dieser Erkenntnis. ${ }^{136}$ Allerdings soll eine Schädigungsabsicht oder ein Bewusstsein der Sittenwidrigkeit nach neuerer Rechtsprechung nicht mehr notwendig sein. ${ }^{137}$ Demgegenüber vertritt die objektive Theorie, dass auch ein objektiver Sittenverstoß allein die Sittenwidrigkeit begründen könne, wenn nicht im Einzelfall die Missbilligung gerade den Zweck des Rechtsgeschäftes trifft. ${ }^{138}$

Der BGH geht in seiner Entscheidung offenbar von der Notwendigkeit des Vorliegens eines subjektiven Tatbestandes im Rahmen des $\S 138$ BGB aus, denn er prüft bei der konkreten Lösung des Falles zunächst ${ }^{139}$ die Gründe für den Ehevertragsschluss in Anlehnung an die Untersuchung der Vorinstanz, wonach die eine Vertragspartei die Unterlegenheit der anderen »missbraucht « habe. Anschließend wendet sich der BGH dem objektiven Gehalt der Vereinbarung zu, um nach der Verneinung der Sittenwidrigkeit sodann zur Ausübungskontrolle überzuleiten mit der Anmerkung, diese sei durchzuführen, wenn sich ergeben habe, dass die von den Parteien getroffenen Abreden »auch in subjektiver Hinsicht « einer Wirksamkeitskontrolle standhalten. ${ }^{140}$ Dieser Ansicht des BGH ist zuzustimmen, ${ }^{141}$ denn das BVerfG hatte für die Inhaltskontrolle gefordert, dass als Folge der Ungleichgewichtslage die einseitige Belastung zustande gekommen sei. ${ }^{142}$ Das Argument für die Inhaltskontrolle war, dass eine Situation vorliegt, in der die Selbstbestimmung in Fremdbestimmung umschlägt. ${ }^{143}$ Solches ist aber

135 BGHZ 94, 268, 272; Palandt/Heinrichs, (FN 59), § 138, Rdz. 7, 46; z.B. die Verpflichtung zur Zahlung eines Entgeltes als Gegenleistung für das Eingehen einer Scheinehe, OLG Düsseldorf, FamRZ 1983, 1023.

136 Staudinger/Sack, (FN 133), § 138, Rdz. 61 m.w.N. zu umfangreicher Rechtsprechung.

137 Staudinger/Sack, (FN 133), § 138, Rdz. 61 m.w.N. zu umfangreicher Rechtsprechung.

138 Staudinger/Sack, (FN 133), § 138, Rdz. 62 m.w.N.; Münchener Kommentar, (FN 132), $\S 138$, Rdz. 130.

139 BGH, NJW 2004, 930 f. = FamRZ 2004, 601 ff. = ZNotP 2004, 157 ff. = DNotZ 2004, 550 ff., Ziffer IV, 1a) der Gründe.

140 BGH, NJW 2004, 930 f. = FamRZ 2004, 601 ff. = ZNotP 2004, 157 ff. = DNotZ 2004, 550 ff., Ziffer IV, 2 der Gründe (noch vor a).

141 Für das Erfordernis eines subjektiven Tatbestandes auch Koch, NotBZ 2004, 147, 148.

142 BVerfG, NJW 1994, 36, 39 und BVerfG, NJW 1994, 2749, 2750.

143 BVerfGE 81, 242, 255; BVerfG, NJW 1994, 36, 38. 
nur möglich, wenn der andere Vertragsteil die Imparität kennt und für den Abschluss des Vertrages nutzt.

Beim zuvor gebildeten Beispiel der schwangeren Verlobten, die sich einer entsprechenden Zwangslage ausgesetzt sieht, ihrem Verlobten aber von der Schwangerschaft nichts gesagt hat, wäre demnach der subjektive Tatbestand beim Vertragspartner zu verneinen. Eine Sittenwidrigkeit läge nicht vor.

Diese Forderung nach Vorliegen subjektiver Voraussetzungen im Rahmen des $§ 138$ BGB kann umso mehr erhoben werden, als ihr Nichtvorliegen die Inhaltskontrolle nicht leer laufen lässt, sondern nur dazu führt, dass die Nichtigkeit nach § 138 BGB zu verneinen ist. Es kann dennoch zu einer Vertragskorrektur mittels Ausübungskontrolle kommen.

Von der Frage des subjektiven Tatbestandes im Rahmen des $\S 138$ BGB ist das Problem des Nachweises zu unterscheiden. Auch diejenigen, welche einen subjektiven Tatbestand fordern, erkennen zum Teil an, dass diese Voraussetzungen nur schwer nachweisbar sind. Aus diesem Grunde wird mehr und mehr aus einer besonders ausgeprägten objektiven Situation auf das Vorliegen des subjektiven Tatbestandes in Form einer widerleglichen Vermutung geschlossen. So löst etwa die BGH-Rechtsprechung nunmehr die Fälle ruinöser Familienbürgschaften, wenn sie eine krasse Überforderung des Bürgen feststellt. ${ }^{144}$ Sie berücksichtigt die individuellen Umstände somit nur noch bei der Widerlegung der Vermutung. Für die Ehevertragsfälle hat so der 15. Deutsche Familiengerichtstag vorgeschlagen, bei einem Globalverzicht das Vorliegen der subjektiven Voraussetzungen widerleglich zu vermuten. ${ }^{145} \mathrm{Ob}$ man allein aus der Prüfungsreihenfolge des BGH, der zunächst nach den subjektiven und dann nach den objektiven Voraussetzungen fragte, schließen kann, eine Vermutung komme für den BGH wohl nicht in Betracht, ${ }^{146}$ ist zweifelhaft, da der BGH nicht wirklich prüfte, sondern nur zu den subjektiven Voraussetzungen bemerkte, diese könne er mangels Sachverhalt nicht prüfen. Dem 15. Deutschen Familiengerichtstag hingegen ist zuzustimmen. Allerdings ist die Vermutung widerlegt, wenn der Verzicht ehetypengerecht erfolgt ist. Denn Maßstab war nicht das dispositive Recht, sondern nur die Grundprinzipien im Lichte des konkreten Ehemodells.

b) Ausübungskontrolle

Liegt nach diesen Kriterien eine Sittenwidrigkeit nicht vor, so kommt die Ausübungskontrolle $^{147}$ als Auffangkontrolle, sozusagen als allgemeine Inhaltskontrolle in Betracht. Hierfür genügt eine typisierbare Fallgestaltung struktureller Unterlegenheit.

Nachdem der BGH die Ausübungskontrolle auch ausdrücklich zulässt als Korrekturinstrument bei nachträglicher Änderung der geplanten Ehekonstellation, kann diese Voraussetzung in solchen Fällen nicht zwingend für den Zeitpunkt des Abschlusses

144 BGH, NJW 2002, 744, 745; Palandt/Heinrichs, (FN 80), § 138, Rdz. 38 f.; vgl. auch schon BGHZ 80, 153, 161.

145 Abschnitt A.III.4., FuR 2004, 18, 20.

146 So mit guter Argumentation Gageik, RNotZ 2004, 295, 307.

147 Gegen Ausübungskontrolle: Enderlein, Rechtspaternalismus und Vertragsrecht, 1996, 414 ff. 
des Ehevertrages gefordert werden. Es muss dann vielmehr auch genügen, wenn eine solche Situation später eintritt. Der BGH beschreibt nicht, wie es später zu einer solchen Situation kommen kann. Er prüft vielmehr, ${ }^{148}$ ob eine Vertragspartei kraft gemeinsamen Entschlusses ein Risiko auf sich nimmt - insbesondere Aufgabe oder Einschränkung der Erwerbstätigkeit -, das sich mit Scheidung zu einem Nachteil verdichtet, und untersucht, ob die Berufung auf die vertragliche Regelung angesichts dessen unbillig ist. Mit dieser Formulierung geht die Rechtsprechung ein Stück in Richtung einer Billigkeitskontrolle, allerdings nicht mit der strengen Konsequenz einer Vertragsnichtigkeit, sondern einer richterlichen Vertragskorrektur. Um im Bild der falltypischen strukturellen Unterlegenheit zu bleiben, könnte man an dem Gedanken der Vertragspflege ansetzen. Wenn ein solches - zuvor nicht abzusehendes Risiko nur von einer Vertragspartei zu tragen ist, so wäre der Ehevertrag ggf. deswegen abzuändern gewesen. Hahne spricht von einem konkludent anderweitigen Vertragswillen, der aus dem faktischen Verhalten ablesbar sei. ${ }^{149}$ Hier ist diejenige Vertragspartei, welche das Risiko auf sich nimmt, von der Vertragssituation her stets im Nachteil, denn sie erreicht diese Abänderung häufig nur schwerlich.

War das betroffene Risiko allerdings bei Vertragsschluss bereits absehbar und hat diejenige Vertragspartei, welche nun benachteiligt ist, das Risiko bewusst auf sich genommen, ohne dass eine Ungleichgewichtslage vorliegt, dann sind die allgemeinen Voraussetzungen einer Inhaltskontrolle insoweit nicht gegeben. ${ }^{150}$ Dies spricht entscheidend dafür, die Vorstellungen der Ehegatten im Ehevertrag detailliert wiederzugeben.

\section{Weitere Perspektiven}

\section{Keine Weiterung zum Recht auf gleiche Teilhabe}

Ist mit der Inhaltskontrolle von Eheverträgen nunmehr ein Instrument gefunden, das der Vertragsgerechtigkeit auf diesem Felde Bahn bricht und eine flexible Kontrolle mit nur ausnahmsweiser Nichtigkeit ermöglicht, so erscheinen Rufe, der BGH habe die Vorgaben des BVerfG nur teilweise umgesetzt und müsse daher mit erneuten verfassungsgerichtlichen Anstößen rechnen, ${ }^{151}$ als zu weitgehend. Denn der vom BVerfG im Urteil zur Ehevertragsfreiheit betonte Grundsatz der gleichberechtigten Partnerschaft bezieht sich gerade auf die Verhandlungssituation und meint, dass die Verhandlungen über das Ehemodell und die dazu passenden Ehescheidungsfolgen im Geiste gleichberechtigter Partnerschaft erfolgen und nicht unter Ausnutzung von Ungleichgewichtslagen. ${ }^{152}$

148 BGH, NJW 2004, 930 f. = FamRZ 2004, 601 ff. = ZNotP 2004, 157 ff. = DNotZ 2004, $550 \mathrm{ff}$. Ziffer IV, 2a) der Urteilsgründe.

149 Hahne, DNotZ 2004, 84, 95.

150 A.A. Dauner-Lieb (FN 105), 1, 26, wo eine eigenständige Ausübungskontrolle vertreten wird, die auch Verträge trotz solcher Risikozuweisungen ändern könne.

151 Rakete-Dombek, NJW 2004, 1273, 1277; kritisch auch Dauner-Lieb, FF 2004, 65, 67 und Grziwotz, FamRB 2004, 199, 239, 241.

152 So auch Rauscher, DNotZ 2004, 524, 553. 
Auch die in der Entscheidung des BVerfG zum Anrechnungsverfahren im Unterhaltsrecht ${ }^{153}$ ausgesprochene Sentenz, dass beide Ehegatten grundsätzlich auch nach Scheidung Anspruch auf gleiche Teilhabe am Erwirtschafteten haben, das ihnen zu gleichen Teilen zuzuordnen sei, und zwar hinsichtlich Unterhalt, ${ }^{154}$ Versorgungsausgleich und Aufteilung gemeinsamen Vermögens, wird mit der Voraussetzung eingeleitet: »Sind die Leistungen, die Ehegatten im gemeinsamen Unterhaltsverband erbringen, gleichwertig...« Daraus folgert der BGH zu Recht, § 1360 Satz 2 BGB meine mit der Gleichwertigkeit nur die Unterhaltsbeiträge. Zudem hat das BVerfG nur zur gesetzlichen Rechtslage Stellung genommen, aber nicht zur Abdingbarkeit durch Vertrag. Außerdem hat sich erwiesen, dass das dispositive Eherecht gerade keinen Leitbildcharakter aufweist, sondern den Ehegatten die Wahl ihres Ehemodells freisteht. Daher ist die Aussage des BVerfG nicht ohne weiteres im Sinne einer strikten Halbteilung auf ehevertragliche Gestaltungen zu übertragen.

\section{Verfassungsrechtlicher Auftrag an den Gesetzgeber $-\S 1585 \mathrm{c} B G B$}

Die Darstellung hat ferner ergeben, dass mit der Möglichkeit formlosen Verzichts auf nachehelichen Unterhalt auch im Kernbereich der Gesetzgeber seinem grundgesetzlichen Schutzauftrag nicht ausreichend nachgekommen ist. Er wird daher aufzurufen sein, hier wie bei den anderen Gegenständen des Ehevertrages den Verzicht nur formgebunden zuzulassen und ggf. zusätzlich Grenzen für einen Verzicht zu entwickeln, der das Kindeswohl beeinträchtigt. ${ }^{155}$

\section{Zusammenfassung}

Die Darstellung der Besonderheiten familienrechtlicher Inhaltskontrolle lässt sich so zusammenfassen:

1. Die Inhaltskontrolle von Eheverträgen ist Missbrauchskontrolle, nicht aber Leitbildabweichungskontrolle, da es an einem gesetzlichen Eheleitbild fehlt und auch Art. 6 Abs. 1 GG mit Ausnahme weniger Strukturprinzipien frei ist von inhaltlichen Vorgaben. Es besteht daher kein allgemeiner Halbteilungsgrundsatz.

2. Maßstab für die Inhaltskontrolle von Eheverträgen sind:

- die vom BVerfG hervorgehobenen Grundprinzipien gleichberechtigter Partnerschaft und unterhaltsrechtlicher Verantwortung aufgrund Elternschaft,

- das unter Respektierung dieser Grundlage von den Ehegatten gewählte Ehemodell.

Die richtige Fragestellung muss also lauten, ob die getroffenen Regelungen der gewählten Ehekonstellation entsprechen. ${ }^{156}$

153 BVerfG, FamRZ 2002, 527, 529.

154 Zur Kritik an dem Begriff der Teilhabe im Unterhaltsrecht: Schwab, FF Sonderheft 2/2004, 164, 171.

155 So nunmehr Referentenentwurf des BMJ, FamRZ 2005, 1041, 1043.

156 Vgl. Dauner-Lieb, FF 2004, 65, 66. 
3. Eheverträge unterliegen nur bei einer Ungleichgewichtslage der Inhaltskontrolle. Dies gebieten Privatautonomie, Rechtssicherheit und die Selbstverantwortlichkeit der Ehegatten, die zu den Grundprinzipien der Ehe gehört.

4. Die Sittenwidrigkeit eines Ehevertrages (Wirksamkeitskontrolle) ist an den allgemeinen Maßstäben des $§ 138$ BGB zu messen, der allerdings grundrechtsgeleitet zu interpretieren ist. Danach ist neben der evident einseitigen Belastung eine objektive Ungleichgewichtslage und subjektiv zumindest die Kenntnis derjenigen Tatumstände erforderlich, welche die Sittenwidrigkeit begründen. Aus einer besonders ausgeprägten objektiven Situation kann auf das Vorliegen des subjektiven Tatbestandes in Form einer widerleglichen Vermutung geschlossen werden. Die Vermutung ist widerlegt, wenn der Vertrag ehetypengerecht ist. Die Eheschließung selbst und die dadurch erreichten Vorteile können dem Verzicht nicht mehr gegenübergestellt werden.

5. § 242 BGB ermöglicht eine Inhaltskontrolle von Eheverträgen im Vorfeld des $\S 138$ BGB und bei nachträglichen Änderungen der Ehekonstellation. Auch hierzu ist, sofern auf den Zeitpunkt des Vertragsschlusses abgestellt wird, eine typisierte Fallgruppe struktureller Unterlegenheit festzustellen. Dieses gebieten die Rechtssicherheit und die grundrechtlich geschützte Privatautonomie. Wurde das später eingetretene Risiko von den Vertragsparteien vorhergesehen und wird im Bewusstsein dessen der Vertrag ohne Ungleichgewichtslage geschlossen, so scheidet eine Änderung durch Ausübungskontrolle aus, außer sie geschieht um des Kindeswohles willen. 